\title{
Phase Evolution During Synthesis of Nanocrystalline Multicomponent (Co,Cu,Mg,Ni,Zn)O Metal Oxides with Varying ZnO Content
}

\author{
Nandhini J. Usharani, Nanta Kumar R., S. S. Bhattacharya \\ Nano Functional Materials Technology Centre, Department of Metallurgical and Materials Engineering \\ Indian Institute of Technology \\ Madras, Chennai - 600 036, India \\ mm14d405@smail.iitm.ac.in; mm13b026@smail.iitm.ac.in; ssb@iitm.ac.in
}

\section{Extended Abstract}

Nanocrystalline ceramics have great potential for applications in electronics, sensors and energy-related areas due to their remarkable functional properties. However, only doped, co-doped and binary ceramics have been extensively studied, while the area of equimolar, multicomponent ceramics has been a largely unexplored field until recently [1]-[3]. In this investigation, a multicomponent nanocrystalline ceramic oxide, $(\mathrm{Co}, \mathrm{Cu}, \mathrm{Mg}, \mathrm{Ni}, \mathrm{Zn}) \mathrm{O}$, was synthesised with the primary intention of studying the type and stability of the phases formed with systematically varying zinc oxide content. The individual components were selected on the basis of Pauling's rules in order to maximise the probability of single phase formation. All these elements have a +2 oxidation state in their stable oxide form. While the oxides of cobalt, magnesium and nickel have a stable rocksalt crystal structure, zinc oxide stabilizes in the wurzite structure and copper has a stable monoclinic structure (a distorted rocksalt structure, due to the Jahn-Teller effect). Therefore, it could be expected that copper and zinc would distribute themselves in the stable lattice structure of the other rocksalt oxides and form a solid solution within the 5 component system, even though it has been reported that in the binary (Ni,Cu)O system, the dominant Jahn-Teller effect leads to the formation of a distorted cubic structure [4].

Based on this premise, a bottom-up, nebulised spray pyrolysis (NSP) approach was selected for synthesis. NSP is a relatively rapid process with adequate residence time which yields clean and stable equilibrium (or near-equilibrium) phases of the product. The process is also industrially scalable. Nitrates of the selected cations were used as precursors and the individual precursor quantities were adjusted in order to maintain the requisite final compositions with de-ionised water as the solvent. X-ray diffraction (XRD) of the as-synthesized powders confirmed the presence of single phase cubic rocksalt structure in the $\mathrm{fm} \overline{3} \mathrm{~m}$ space group for all the compositions. The variation of the synthesis temperature shows a decreasing trend from $1400{ }^{\circ} \mathrm{C}$ to $1100{ }^{\circ} \mathrm{C}$ for uniform increase in the concentration of $\mathrm{ZnO}$ from $4 \%$ to equimolar composition because of increasing configurational entropy towards equimolar concentrations. This observation could be also due to the decrease in Jahn-Teller effect with decrease in $\mathrm{CuO}$ concentration. The crystallite size calculated using Scherrer formula shows a decrease from $48 \mathrm{~nm}$ for $4 \% \mathrm{ZnO}$ to $20 \mathrm{~nm}$ for $20 \% \mathrm{ZnO}$ in a linear fashion since $\mathrm{ZnO}$ has a different crystal structure, necessitating more energy requirement to dissolve $\mathrm{ZnO}$ in rocksalt lattice leaving lesser energy for crystallite growth. Also with increasing $\mathrm{ZnO}$ concentration, its dissimilar crystal structure hinders the diffusion of isostructured components leading to lesser crystallite growth.[5]. Scanning electron microscopy (SEM) revealed the particles to have broken shell like morphology and energy dispersive spectroscopy associated with the SEM confirmed the composition.

\section{References}

[1] A. Sarkar, R. Djenadic, N. J. Usharani, K. P. Sanghvi, V. S. K. Chakravadhanula, A. S. Gandhi, H. Hahn, S. S. Bhattacharya, "Journal of the European Ceramic Society Nanocrystalline multicomponent entropy stabilised transition metal oxides," J. Eur. Ceram. Soc., vol. 37, no. 2, pp. 747-754, 2017.

[2] R. Djenadic, A. Sarkar, M. Botros, C. Loho, O. Clemens, V. S. K. Chakravadhanulau, C. Kübel, S. S. Bhattacharya, A. S. Gandhi, H. Hahn, "Multicomponent equiatomic rare earth oxides," Phys. status solidi - Rapid Res. Lett., vol. 
$3831,2016$.

[3] C. M. Rost, E. Sachet, T. Borman, A. Moballegh, E. C. Dickey, D. Hou, J. L. Jones, S. Curtarolo, J.-P. Maria, "Entropy-stabilized oxides," Nat Commun, vol. 6, 2015.

[4] P. K. Davies and A. Navrotsky, "Thermodynamics of solid solution formation in NiO-MgO and NiO-ZnO," J. Solid State Chem., vol. 38, no. 2, pp. 264-276, 1981.

[5] N. Y. C. Xu, J. Tamaki, N. Miura, "Stabilization of $\mathrm{SnO}_{2}$ ultrafine particles by additives," vol. 27, pp. 963-971, 1992. 